http://dx.doi.org/10.4314/gjl.v7i2.2

\title{
OPTIMAL INFLECTIONS AS ASYMMETRY BETWEEN NOMINAL AND VERBAL REDUPLICATIONS IN AKAN
}

\author{
Charles Marfo and E. Kweku Osam
}

\begin{abstract}
This paper discusses two issues in nominal and verbal reduplications in Akan, a language which is widely spoken in Ghana. These are the respective morphotactic structures of the two reduplications and the claim that an asymmetry obtains between nominal and verbal reduplications in the language. The issues are discussed in connection with a distinction in inflection for the nasal prefix $/ \mathrm{N}-/$ in nominal and verbal reduplications of Akan which, respectively, impute negation and plurality and how the individual inflections underscore and inform the morphotactic structures of the two reduplications. Analysis of issues will be done in the light of the Morphological Doubling Theory (Inkelas and Zoll 2005, Osam et al. 2013) and will further be captured within Optimality Theory (e.g. Prince \& Smolensky 2004, McCarthy \& Prince 1999). The discussions will particularly be narrowed down on the more interesting morphotactics of the verbal reduplication and its inflection for other verbal affixes (besides /N-/). Establishing the suggested asymmetry, we will also endeavour to show the general order of inflection in the verbal reduplication as opposed to what obtains in the nominal reduplication following structural well-formedness in both reduplicated forms.
\end{abstract}

Keywords: Reduplication, inflection, morphotactics, asymmetry, doubling, constraints, well-formedness 
Marfo \& Osam: Optimal Inflections as Asymmetry Between Nominal and Verbal Reduplications in Akan

\section{Introduction}

This paper looks at reduplication in Akan, a Kwa language widely and chiefly spoken in Ghana. ${ }^{1}$ Works on Akan reduplication include Dolphyne (1988), Winkler and Obeng (2002), and Osam et al. (2013). Generally, these works have mainly described the phenomenon with some morphophonological and semantic overtures. In this paper, however, besides the descriptions where necessary, the discussion is devoted to two issues regarding nominal and verbal reduplications in Akan. These are: i) the respective morphotactic structures of nominal and verbal reduplications in the language, ii) the claim that there obtains an asymmetry between respective morphotactic structures of the two reduplicated forms. As will become evident in the course of the discussions, these two issues are actually intertwined in the sense that the said asymmetry explains a distinction in the inflection for a particular prefix / $\mathrm{N}$-/ between the nominal and verbal reduplicated forms in Akan and, therefore, the respective morphotactic structures.

Furthermore, the discussions will be narrowed down to the more interesting verbal reduplication, where we will endeavour to establish the general morphological order with respect to the inflection for $/ \mathrm{N}-/$ and other verbal affixes. Particularly, the inflection for the affix $/ \mathrm{N}$-/ in verbal reduplication will be observed against what happens when the affix is also inflected for in nominal reduplication. The essence is to underscore individual morphotactic structural well-formedness for/between the nominal and the verbal reduplicated forms in Akan and to emphasize the asymmetry we contend obtains between these two reduplications with respect to how the affix is inflected for in each case.

\subsection{Reduplication}

Among other definitions, reduplication has generally been described as a morphological process involving systematic recurrence of a unit within a word for semantic or grammatical purposes (e.g. Marantz 1982, Rubino 2005, and Inkelas 2005). As the data in (1) exemplify, reduplication occurs in all the major word classes in Akan. In defining reduplication, Haspelmath (2002: 274) dwells on the formal changes associated with reduplicated words. He defines it as "a morphological process which repeats the morphological base either entirely or partially". These are respectively shown in (i)-(iv) and (v) of (1).

\footnotetext{
${ }^{1}$ Akan includes Asante, Akuapem, Fante, Bono, and Akyem. In this paper, data is mainly taken from Asante.
} 
(1)

\begin{tabular}{|c|c|c|c|}
\hline Noun: & i. & $\begin{array}{l}\text { Basic form } \\
\text { dua 'piece of wood' } \\
\text { kaseq 'bone/thorn' }\end{array}$ & 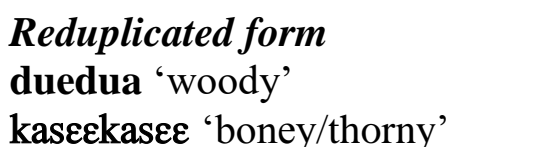 \\
\hline Adjective: & ii. & 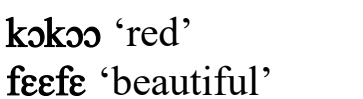 & 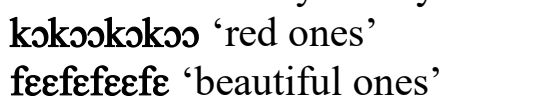 \\
\hline Adjective: & iii. & 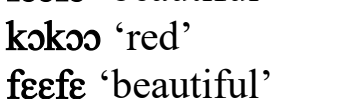 & kokokoko 'very red' \\
\hline Adverb: & iv. & $\begin{array}{l}\text { bokso 'slow' } \\
\text { dinn 'quiet' }\end{array}$ & $\begin{array}{l}\text { bokobokos 'slowly' } \\
\text { dinndinn 'quietly' }\end{array}$ \\
\hline Verb: & V. & $\begin{array}{l}\text { horo 'to wash/clean' } \\
\text { kyere 'to tie' }\end{array}$ & $\begin{array}{l}\text { hohoro 'to wash (extensively)' } \\
\text { kyekyere 'to tie (extensively)' }\end{array}$ \\
\hline
\end{tabular}

Specifically, in (i)-(iv) of (1), we observe a total reduplication in the sense that the stem is repeated although in (iii) there is some sort of vowel elision in one part or the other in the stem of the adjective. In (v), on the other hand, the succeeding syllable of the stem is strictly elided and preceded to the basic form (as the reduplicant), hence a partial reduplication. ${ }^{2}$

\subsection{Theoretical framework}

Analysis of the structures of nominal and verbal reduplications will be done with recourse to stipulations of the Morphological Doubling Theory (Inkelas 2005, Inkelas and Zoll 2005, Osam et al. 2013, etc.) which, among other assumptions, critically suggests that daughters in a reduplication (i.e. reduplication members) are semantically identical. For the purposes of achieving clarity in the nominal and the verbal reduplication structures and how they are individually constrained, which contributes to a comprehensive presentation of structure, the analysis will further be couched within Optimality Theory (e.g. Prince \& Smolensky 2004, McCarthy \& Prince 1999), a linguistic machinery which proposes that the heart of grammar lies in the interaction of universal constraints.

\footnotetext{
${ }^{2}$ The partial reduplicated forms given in (v) of (1) could further be reduplicated as well. In this wise, total reduplication is realized; i.e. 'hohoro' becomes hohorohohoro 'to wash several times or several places' and kyekyere becomes kyekyerekyekyere 'to tie several times or several places'. It is also interesting to note that the so-called basic forms of 'horo' and 'kyere' could actually be said without the last syllable in Asante-Twi; i.e. ho and kye respectively.
} 
The rest of the paper is organized as follows: Section 2 looks at reduplication in Akan, focusing on the two types that are of interest in this paper - the nominal and verbal reduplications. The inflection of the prefix $/ \mathrm{N}-/$ in nominal and verbal reduplications is discussed in section 3, where some comparison is also done. In section 4, the Morphological Doubling Theory, as our theory of analysis, is employed to examine the individual structures of the nominal and verbal reduplications. Along with optimality theoretic analysis, the theory will also be explored to establish the asymmetry that is suggested to obtain between the individual morphotactic structures of the nominal and verbal reduplications. Section 5 concludes the paper.

\section{Reduplication in Akan}

As noted in section 1.1, reduplication is a morphological process and occurs in all the major words classes - i.e. nouns, verbs, adjectives and adverbs - in Akan. In fact, it obtains in idiophones in the language as well; e.g kyakaa 'of a wetly ground' is reduplicated as kyakakyaka. In terms of the structure of reduplication, Osam et al. (2013) show that a verbal reduplication is constructed via an extension to the left of the morphological base of the verb in the case of Akan. They, therefore, describe reduplication as left-directed and this seems to obtain in the nominal reduplication of Akan as well. With examples from total reduplication, they explain that the left direction of reduplication in Akan is due to the fact that, where the reduplication members are phonetically different, the morphological base is the member that reflects the original stem; i.e. the phonetic form of the base. The other then becomes the reduplicant.

In both the nominal and verbal reduplications, we observe that the succeeding reduplication member reflects the stem that undergoes reduplication, as shown in (2a). Therefore, it is assumed to constitute the base and the structure is captured as 'CONSTITUENT ${ }_{\text {ReduPlicant }}^{1}$-CONSTITUENT ${ }^{2}{ }_{\text {BASE }}$ ' $\left(\mathrm{C}^{1}{ }_{\text {RED }}-\mathrm{C}^{2}{ }_{\text {BASE }}\right)$. The 'left-directed' argument in the reduplication is further enforced by what happens in the case of verbs that undergo partial reduplication. As could be observed in (2b), a recast of (v) of (1) for ease of reference, it is important to note that it is the base that reflects the basic stem of the verb, with the reduplicant partially reflecting the base. 
(2) $\quad \mathrm{a}$

a

$$
\begin{array}{ll}
\text { Noun: } & \text { i. } \begin{array}{l}
\text { dua 'piece of wood' } \\
\text { sika 'money/gold' }
\end{array} \\
\text { Verb: } & \text { ii. } \begin{array}{l}
\text { tua 'to pay' } \\
\text { hye 'to wear/arrange' } \\
\text { bo 'to hit/break' }
\end{array}
\end{array}
$$

$\mathrm{b}$
Reduplicated form $\left(\mathrm{C}^{1}{ }_{\mathrm{RED}}-\mathrm{C}^{2}{ }_{\mathrm{BASE}}\right)$

[duedua] 'woody'

[sikesika] 'golden/like gold'

[tuetua] 'to pay (more than one item)'

[hylhys] 'to wear (one after another)'

[bobo] 'to hit/break (one after another)'
Basic form

Verb: i. horo 'to wash/clean'

ii. kyere 'to tie'
Reduplicated form $\left(\mathrm{C}_{\mathrm{RED}}^{1}-\mathrm{C}^{2}{ }_{\mathrm{BASE}}\right)$

hohoro 'to wash (extensively)'

kyekyere 'to tie (extensively)'

Let us note that the change in the phonetic form of the vowel at the right-edge of each reduplicant in (2a) (i.e. [e] in '[[due]dua]'; [I] in '[[hyr]hye]' and [v] in [[bo]bs]) is due to a phonological rule that requires a rise in the vowel height to the immediately preceding height relative to the corresponding one in the base. From the Akan vowel chart given in (3) and the vowel harmony principle in Akan, ${ }^{3}$ therefore, the rise from [a] to [e]; [ع] to [I] and [o] to $[\mathrm{U}]$ as indicated by the arrows is understandable. That is, the advanced (i.e. [+ATR]) vowel /a/ rises to /e/ rather than its immediately preceding high $/ \varepsilon /$ because $/ \varepsilon /$ is an unadvanced (i.e. $[-\mathrm{ATR}]$ ) vowel and could not have harmonized with the vowels in the base, hence the preference for /e/, the next preceding high, which is also [+ATR]. The same explanation goes for rest.

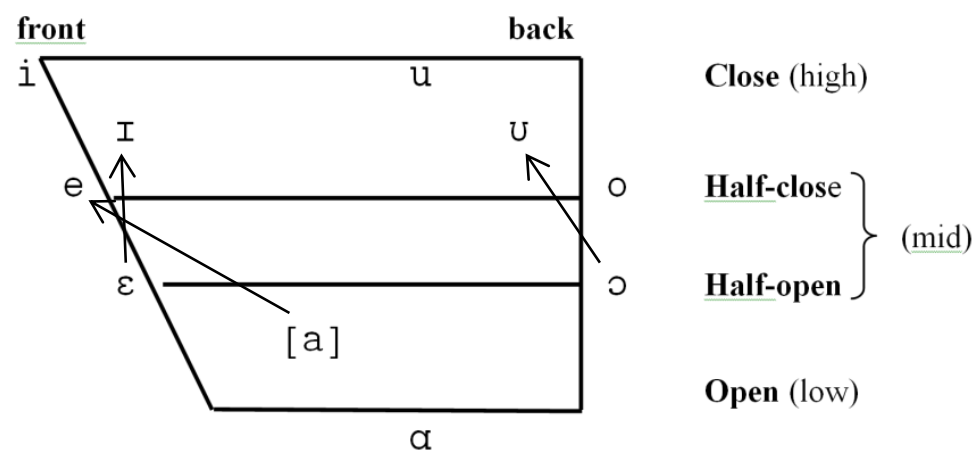

\footnotetext{
${ }^{3}$ Observe that, in addition to the vowel heightening, the advancement of the tongue root (ATR) vowel harmony must apply. As a regressive rule in Akan, the spreading of [+/-ATR] feature is initiates from the stem-final vowel of the constituent at the right-edge and that can only be in the morphological base - i.e. $\left[\mathrm{C}_{\mathrm{RED}}^{1}-\mathrm{C}^{2}{ }_{\text {BASE }}\right]^{\mathrm{ATR}}$. See Osam et al. (2013) for details.
} 

in Akan

One may describe what we strive to do as a mere comparative study between nominal and verbal reduplications. However, considering the differences in inflection for the affix $/ \mathrm{N}-/$ between the two reduplications, in this paper, we strive to establish a reduplication asymmetry between them as well. In this respect, asymmetry is explained in terms of the morphemes that undergo reduplication in the nominal and verbal reduplications and, for that matter, how reduplication is individually realized. Specifically, we show in the following sections that $/ \mathrm{N}-/$ as a nominal affix reduplicates along with its host, whereas $/ \mathrm{N}$ / as a verbal affix does not duplicate along with its host. In effect, what matters here is not that we have two different word classes, but the fact that these two different word classes both inflect for the affix $/ \mathrm{N}-/$, which imputes different grammatical forms and, for that matter, designates two different morphemes; i.e. number in nouns and negation in verbs. Sato (2009) looks at a similar asymmetry in reduplication in Bahasa Indonesia and claims that verbal affixes allow only stem reduplication, whereas nominal affixes allow both stem and stem-affix reduplication in the language.

\subsection{Nominal Reduplication in Akan}

Semantically, the reduplication of nouns immediately imputes plurality. Following Dolphyne (1988: 136), Boakye (2015: 26) makes two observations in connection with nominal reduplication in Akan. First, he observes that only nouns in their plural forms may be reduplicated. Secondly, he notes that the reduplication is usually total in nature as it basically involves a repetition of the (plural) noun; i.e. the reduplicant is a copy of the base. These could be observed from the data in (4). On first observation, the unstated suggestion from Dolphyne (1988) and Boakye (2015) is that reduplication of nouns in the singular would be ill-formed. However, as could be seen in (5) our current data show otherwise; there are cases where nouns in the singular are reduplicated. We acknowledge, however, that nominal reduplication in Akan generally occurs with the plural forms.

$\begin{array}{ll}\text { Noun } & \text { Plural form } \\ \text { dompe 'bone' } & \text { n-nompe } \\ \text { dua 'stick/wood' } & \text { n-nua } \\ \text { a-kwadaa 'child' } & \text { n-kwadaa } \\ \text { e-kuro 'town' } & \text { n-kuro }\end{array}$

Reduplication n-nompe-n-nompe 'of bones/bony' n-nue-n-nua 'woody' n-kwadaa-n-kwadaa 'little children' n-kuro-n-kuro 'several towns' 
Noun

dompe 'bone'

dua 'stick/wood'

a-kwadaa 'child'

e-kuro 'town'
Reduplication

dompe-dompe 'of bone'

due-dua 'of wood'

a-kwadaa- $\varnothing-k w a d a a$ 'of a child'

e-kuro-ø-kuro 'towns'

An interesting observation that would later be further discussed in connection with the matter of asymmetry between nominal and verbal reduplications is the status of the nominal prefix in reduplication. We observe that, unlike in the plural case of nominal reduplication in (4) where there is total reduplication (i.e. both the prefix and the stem are reduplicated), in the singular case, only the stem is reduplicated. Hence, as indicated in (c) and (d) of (5), in particular, no morph $(\varnothing)$ is realized in the reduplicant.

We also observe that nominal reduplications could serve as modifiers, in which case they behave like adjectives. In this modifying role, a nominal reduplication imputes the semantic of content of increase in magnitude. Where this is the case, a nominal reduplication as adjective could appear in both the attributive case as in (6a) and in the predicative case as in (6b).

\begin{tabular}{|c|c|c|}
\hline $\begin{array}{l}\text { i. } \\
\text { ii. }\end{array}$ & $\begin{array}{l}\text { Attributive case } \\
\text { Enam nnompennompe } \\
\text { bankye nnuennua }\end{array}$ & $\begin{array}{l}\text { 'bony meat' } \\
\text { 'woody cassava' }\end{array}$ \\
\hline i. & $\begin{array}{l}\text { Predicative case } \\
\text { Enam no ye nnompennompe } \\
\text { meat DEF. to-be bony } \\
\text { 'The meat is bony.' }\end{array}$ & \\
\hline ii & $\begin{array}{l}\text { Bankye no ye nnuennua } \\
\text { cassava Def. to-be woody } \\
\text { 'The cassava is woody.' }\end{array}$ & \\
\hline
\end{tabular}

Furthermore, it is important to point out that some of the nouns in Akan that are often readily subjected to the process of reduplication are what have been referred to in the literature as nominalized nouns (e.g. Appah 2003, Adomako 2015); they are nouns that have been derived from other word classes, particularly verbs and adjectives in the case of 

in Akan

Akan. Respective examples of these are given in (7a) and (7b). It is interesting to note that, when the nominalized (Nom.) form is reduplicated, the semantic content of increase in magnitude is well catered for with the repeated nominal affix /A/, which doubles as the irregular plural affix. ${ }^{4}$

$\begin{array}{ll}\mathrm{a} & \mathrm{i} \\ \mathrm{i} & \mathrm{i} \\ \mathrm{i} & \end{array}$

$\mathrm{b}$

$\begin{array}{ll} & \text { Adjective } \\ \text { i. } & \text { kese 'big/large' } \\ \text { ii. teaa 'slim' } \\ \text { iii. } & \text { kokso 'red' }\end{array}$

\author{
Nom. Form \\ a-pra 'sweeping' \\ a-tea 'punishment' \\ a-wosos 'shaking'
}

Nom. Form

o/a-kese $\varepsilon$ '... of stature'

a-teaa 'slim body'

a-kəkəo 'red ones'

\author{
Reduplication \\ a-pra-a-pra \\ a-tea-a-tea \\ a-woso-a-wosos
}

\author{
Reduplication \\ a-kese $\varepsilon-a-k \varepsilon s e \varepsilon$ \\ a-tea-a-tea

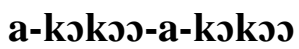

\subsection{Verbal reduplication in Akan}

According to Moravcsik (1978), reduplication of verbs is used to express repetitive or iterative and distributive actions. These expressions have been observed in diverse languages including Akan. In Akan, reduplication of verbs (and some other categories) also expresses intensification and increase in number of one or both of the verb's argument functions. For instance, as could be observed in (8a), where the verb bo 'to hit' is reduplicated into $\boldsymbol{b o b s}$, the reduplication imputes the meaning of repetition and/or intensity in the act of hitting and the fact that the hitting is on different parts of the target/patient. This results in the meaning 'hit repeatedly' or 'beat up'. We also find in (8) the fact that verbal reduplication legitimizes its object in the plural as in (8b) in the sense that the expression of distribution by the verb rightly demands plurality of the object to mean that the hitting is distributed among many patients; i.e. nkwadaa 'children'. Likewise, in (8c), the expression of group action by the verb rightly explains the plurality of the subject to mean that the hitting is not done by one person, but two or more; i.e. mmaa 'women'.

\footnotetext{
${ }^{4}$ Let's emphasize that the derivation is marked by the nominal prefix /A-/ or /O-/ in the singular and / N-/ in the common or regular case of plurality and where explicit expression of increase in magnitude is possible. In (7a) and (7b), some of these derived nouns, the class of their source - i.e. verb and adjective - before nominalization and the ensuing reduplicated forms could be observed. See Section 4 for further insight.
} 
(8) a. Kofi bobo-s a-kwadaa no

PN. RED.hit-PAST child.Sing. Det.

'Kofi beat up the child'.

b. Kofi bobo-s $n$-kwadaa no

PN. RED.hit-PAST child.Plu. Det

'Kofi beat up the children'.

c. Mmaa no bobs-s a-kwadaa no

woman.Pl. Det. RED.hit-PAST child.Sing. Det

'The women beat up the child'.

*d. Kofi bo-s n-kwadaa no

PN. hit-PAST child.Plu. Det

'Kofi beat the children'.

*e. Mmaa no bo-s n-kwadaa no

Woman.Pl. Det. hit-PAST child.Plu. Det

'Kofi beat the children'.

If we compare (8b) to (8d) and (8c) to (8e), we observe that, without verbal reduplication, (8d) and (8e) are ungrammatical. That is, respectively, many patients could not have suffered a single punch and many agents could not have given a single punch and, also, to a number of patients. Let us also note that, unlike in the case of nominal reduplication where many nouns and derived ones require the nominal affix for reduplication, the verb needs no inflection before reduplication takes place. Accordingly, as the data in (9) exhibit, the morphological base could immediately be reduplicated.

(9)
Verb
Reduplication
a. pra 'sweep'
pra-pra 'sweep (around)'
b. kasa 'speak'
c. dua 'plant'
kasa-kasa 'speak scornfully'
d. pam 'sew'
due-dua 'plant (around)'
pim-pam 'sew (here and there)' 


\section{Inflection for $/ \mathrm{N}-/$ as a factor of asymmetry}

We have noted that the morphological bases of both the noun and the verb inflect for the prefix /N-/ for different semantic inputs. While the noun inflects for the affix $/ \mathrm{N}-/$ for plurality, the verb inflects for it to negate its affirmative form. These are respectively exemplified in (10a) and (10b) below, where the phoneme $/ \mathrm{N}-/$ is variously realized according to the place of articulation of the stem-initial consonant. Our major interest, however, is in the inflection for the affix in the individual reduplications of the noun and the verb because, as we have noted earlier and will attempt to evince, the individual morphological orders reveal an asymmetry between the nominal and verbal reduplications.

$\begin{array}{ccll}\text { a } & \text { Noun } & \begin{array}{l}\text { Plural form } \\ \text { dompe 'bone' }\end{array} & \text { n-nompe } \\ & \text { m-moa } \\ & \text { ii. } & \text { a-boa 'animal' } \\ \text { iii. } & \text { a-fenaa 'servant' } & \text { m-fenaa [mfinaa] } \\ & \text { iv. } & \text { e-kuro 'town' } & \text { n-kuro [nkuro] } \\ & & & \\ \text { b } & & \text { Verb } & \text { Negative form } \\ & \text { i. } & \text { sa 'dance' } & \text { n-sa } \\ & \text { ii. } & \text { boa 'help' } & \text { m-moa } \\ & \text { iii. } & \text { fra 'mix', } & \text { m-fra [mfra] } \\ & \text { iv. } & \text { kum 'kill' } & \text { n-kum [nkum] }\end{array}$

With the nouns, it must be emphasized that their inflection for $/ \mathrm{N}-/$ even before reduplication is for the regular manifestation of plurality as shown in (10a). As also noted and shown in (11a), we observed that a few other nouns in the language rather take an alternative plural prefix of /A-/, often described as the irregular case. Yet others, often described as mass or countless nouns, are also noted with the prefix $/ \mathrm{N}-/$ in their citation forms and proceed to be reduplicated as shown in (11b).

$\begin{array}{cll}\text { a } & \text { Noun } & \text { Plural form } \\ \text { i. } & \text { bosom 'idol' } & \text { a-bosom 'idols' } \\ \text { ii. } & \boldsymbol{\varepsilon} \text {-dan 'house' } & \text { a-dan 'houses' } \\ \text { iii. } & \text { E-kwan 'way' } & \text { a-kwan 'ways' } \\ \text { iv. } & \text { e-tuo 'gun' } & \text { a-tuo 'guns' }\end{array}$

Reduplication a-bosom-a-bosom 'various idols' a-dan-a-dan 'a number of houses' a-kwan-a-kwan 'road after road' a-tuo-a-tuo 'range of guns' 
b

$$
\begin{array}{ll}
\text { i. } & \text { n-suo 'water' } \\
\text { ii. } & \text { n-kyene 'salt' } \\
\text { iii. } & \text { m-mogya 'blood' } \\
\text { iv. } & \text { m-paee 'prayer' }
\end{array}
$$

Reduplication

n-suo-n-suo 'watery'

n-kyene-n-kyene 'salty'

m-mogya-m-mogya 'of bloodstain'

m-pae $\varepsilon-\mathrm{m}-$ pae $\varepsilon$ 'prayer after prayer'

With the verb, on the other hand, the inflection for the prefix / $\mathrm{N}-/$ is not needed at all for reduplication as we observed in section 2.2. However, as could also be observed in (12), where the prefix $/ \mathrm{N}-/$ is inflected for in reduplication, the imputation of negation is evident just as it is with its inflected morphological stem.
Verb
Neg-Stem
Neg-Reduplication
a. pra 'sweep'
m-pra
m-pra-pra
b. kasa 'speak'
n-kasa
n-kasa-kasa
c. dua 'plant'
n-nua (/n-dua/)
d. fem 'loan out'
$\mathrm{m}$-fem
n-nua-dua
m-fem-fem

On the realization of asymmetry between the nominal and the verbal reduplications, we observe that the morphological stem of the nominal reduplication allows affix-stem reduplication pattern (i.e. [[Affix-Stem]-[Affix-Stem]]), but that of the verbal reduplication blocks this pattern, but rather allows only stem reduplication (i.e. [Affix-[[Stem]-[Stem]]]), hence the asymmetry (a) and (b) of (13) below respectively shows.

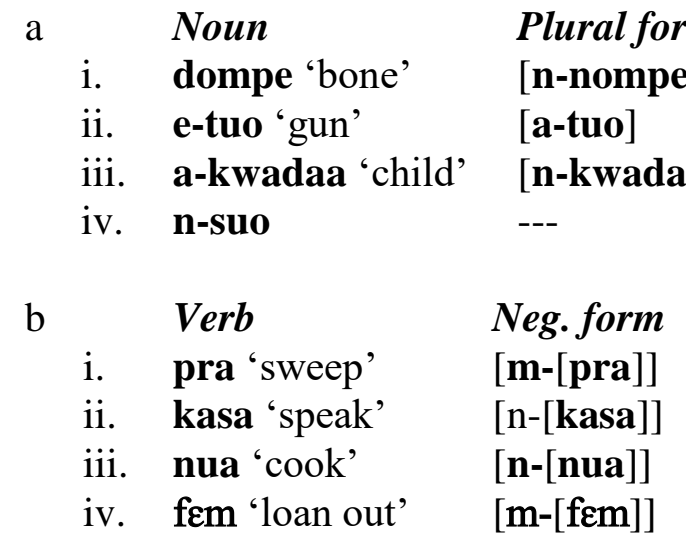

Reduplication

[[n-nompe]-[n-nompe]]

[[a-tuo]-[a-tuo]]

[[n-kwadaa]-[n-kwadaa]]

[[n-suo]-[n-suo]]

$\begin{array}{ll}\text { Reduplication } & * \text { Reduplication } \\ [\text { m-[pra }]-[\text { pra }]] & *[[\text { m-pra }]-[\text { m-pra }]] \\ {[\text { n- }[\text { kasa }]-[\text { kasa }]]} & *[[\text { n-kasa }]-[\text { n-kasa }]] \\ {[\text { n- }[\text { nua }]-[\text { nua }]]} & *[[\text { n-nua }]-[\text { n-nua }]] \\ {[\text { m- }[\text { fem }]-[\text { fem }]]} & *[[\text { m-fem }]-[\text { m-fem }]]\end{array}$



in Akan

The data in (13) may be explained further through prosodic considerations of domain categorization (see e.g. Selkirk (1986) and de Lacy (1997)), and we postulate that the morphological stem of the noun is prosodically dependent on the inflected affix for domain sufficiency and/or requisition for reduplication. ${ }^{5}$ We further suggest that this requisition explains why the noun stem in Akan generally inflects for the plural affix before reduplication can be realized. Accordingly, as structured in (13a) under 'reduplication' in particular, this dependence ensures the realization of a true total reduplication (i.e. [[AffixRED]-[Affix-BASE]]) in nominal reduplication. In the case of the verbal reduplication, on the other hand, we contend that the morphological stem is prosodically sufficient and, therefore, independent (of the affix $/ \mathrm{N}-/$ ). Accordingly, unlike the noun, it could immediately be reduplicated in isolation and without the prefix as the data in (13b) illuminates. That is to say, as shown under 'reduplication 2' of (13b), verbal reduplication involving the base and the affix is ill-formed.

Subsequent to the prosodic independence of the morphological base of the verb into reduplication, we posit that where we have affix in verbal reduplication as in (13b), it only comes in or it is inflected for after reduplication of the stem. This explains why the affix is outside the immediate domains of the base and its reduplicant under 'reduplication'; [Affix-[[RED]-[BASE]]]. The data in (14) further explain the fact that, unlike the nominal base, the verbal base does not need the affix for reduplication.
Verb
Reduplication
a. pra 'sweep'
pra-pra 'sweep around'
b. kasa 'speak'
c. dua 'plant'
kasa-kasa 'speak scornfully'
due-dua 'plant (around)'
d. pam 'sew'
pim-pam 'sew (here and there/one after the other)'

The fact that the verbal base is prosodically sufficient for reduplication is buttressed by the fact that other affixes it inflects for, particularly tenses and/or aspects, are also not individually inflected for by the reduplicant and the base. As shown in (15), we observe a general inflection for the affixes and not an individual inflection of them by the reduplicant and the base (i.e. *[[Affix-RED]-[Affix-BASE]]), as also shown in (15).

\footnotetext{
${ }^{5}$ In this paper, we do not assume any particular prosodic domain. All what we need to show is that the morphological base is reduplicated; i.e. either with the affix $/ \mathrm{N}-/$ or in isolation.
} 


\begin{tabular}{|c|c|c|c|}
\hline \multirow[b]{2}{*}{ i. } & Verb & Reduplicated verbs & \\
\hline & pra 'sweep' & Kofi [re-[pra-pra]] & *Kofi [[re-pra]-[re-pra]] \\
\hline \multirow{3}{*}{ ii. } & & $\begin{array}{l}\text { PN PROG.-RED-sweep } \\
\text { 'Kofi is sweeping around' }\end{array}$ & \\
\hline & kasa 'speak' & Kofi [be-[kasa-kasa]] & *Kofi [[be-kasa]-[be-kasa]] \\
\hline & & $\begin{array}{l}\text { PNFUT.RED-speak } \\
\text { 'Kofi will speak scornfully' }\end{array}$ & \\
\hline \multirow[t]{2}{*}{ iii. } & nua 'cook' & Kofi [[due-dua]-e] & $*$ Kofi $[[$ due-e]-[dua-e $]]$ \\
\hline & & $\begin{array}{l}\text { PN RED-plant-PAST } \\
\text { 'Kofi planted (around)' }\end{array}$ & \\
\hline \multirow[t]{3}{*}{ iv. } & fem 'loan out' & Kofi $[\mathbf{a}-[$ fem-fem] $]$ & *Kofi [[a-fem]-[a-fem]] \\
\hline & & PN COMP-RED-loan out & \\
\hline & & 'Kofi has loan out a lot' & \\
\hline
\end{tabular}

\section{Reduplicated forms in Morphological Doubling Theory}

We have observed in section 2 that nominal and verbal reduplications in Akan are mostly total. Reduplication of the noun and the verb, therefore, involves the doubling of the morphological stem as the Morphological Doubling Theory (Inkelas 2005, Inkelas and Zoll 2005, Osam et al. 2013; etc.) suggests. According to Inkelas and Zoll (2005: 2), the central thesis of the Morphological Doubling Theory (MDT) is that the phonological, morphological and semantic aspects are needed and that their empirical domains of application are almost complementary. They explain further that, key to MDT is the assumption that daughters of reduplication are semantically identical. Thus, as shown in (16) below, a recast of Inkelas (2005: 65), a morphological construction calls for two autonomous inputs/stems whose morphosyntactic features are in agreement.

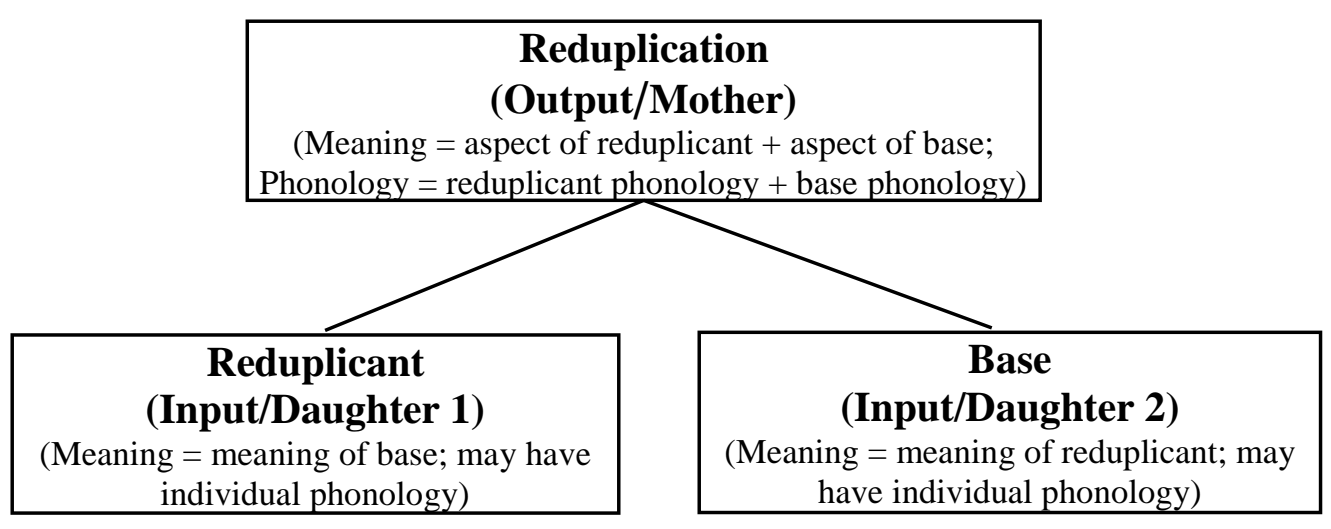



in Akan

We also note in (16) that, while the individual stems do share the same morphosyntactic features, they may be morphotactically and morphophonologically distinct; a morphological divergence that is unique to MDT. So, each input (noted as a daughter) is also subject to a co-phonology that determines its shape. However, the outputs of the two stems' co-phonologies are combined and subjected to a third co-phonology at a combined stage (noted as the mother node) that produces a surface form. In other words, the reduplicant and the base are also subject to a common phonology that determines a resulting shape (or final morphological structure) of an output. Instances of diverse cophonologies are seen in some nominal and verbal reduplications in Akan; e.g. n-nue-nnua (from the stem, edua/nnua 'stick/wood/tree') and $\boldsymbol{d} \boldsymbol{o}-\boldsymbol{d} \boldsymbol{o}$ (from the basic form, $\boldsymbol{d} \boldsymbol{o}$ 'to weed'), respectively. We observe differences in vowel(s) between the daughters, indicating differences in phonology. However, they share a common morphosemantic description.

In the affirmative state of the verb, the outputs of the nominal and verbal reduplications are commonly captured and straightforwardly explained in MDT. ${ }^{6}$ That is, as could be seen in the respective cases of (17a) and (17b) for nominal and verbal reduplications, both inputs (i.e. the reduplicant and the base) are morphophonologically identical. In this case, they are also identical in terms of morphotactics. Accordingly, each one of them is well-formed, resulting in identical order or symmetry. Within optimality theory, the constraints 'MAX-IO' (McCarthy and Prince 1995) as the dominant constraint ensures the doubling or total reduplication in the nominal and verbal cases. Stated in (19a), 'MAX-IO', in this case, outranks any constraint that suggests any morpheme out in the reduplication. One such constraint we employ is 'Truncate', which is also stated in (19b), and ranked lower to 'MAX-IO' in (18).

\footnotetext{
${ }^{6}$ Let us be reminded that, unlike the verb which inflects for $/ \mathrm{N}-/$ to indicate negation and could be reduplicated without the affix, the noun inflects for $/ \mathrm{N}-/$ to indicate plurality and it is generally needed for reduplication.
} 
(17) a

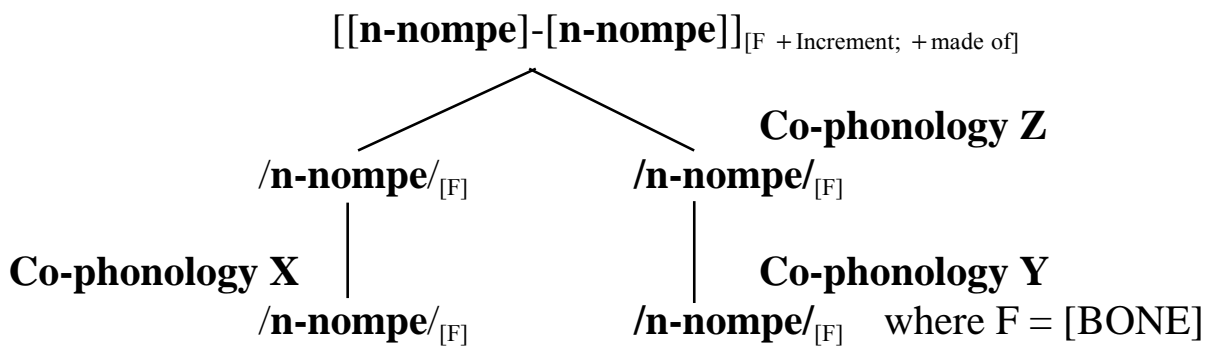

b.

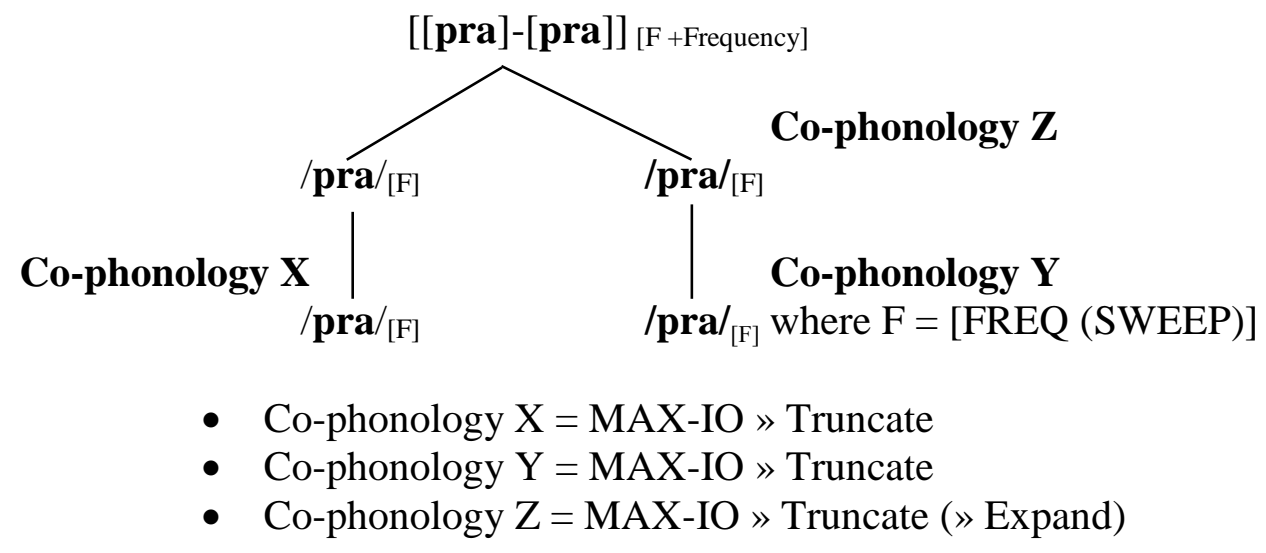

a. MAX-IO: Every element of the input must have a correspondent in the output.

b. Truncate: Truncate where the morphotactic structure of the word demands that.

We further observe in (17a) and (17b) that the co-phonologies ' $\mathrm{X}$ ' and ' $Y$ ' of 'input 1' (i.e. reduplicant) and 'input 2' (i.e. base) share a common constraint ranking (see (i) and (ii) of (17c)) that legitimizes their individual forms, yet identical outputs, which then become the inputs for evolving the main output; i.e. the reduplication. This reduplication also emerged on co-phonology ' $Z$ ' (with the same ranking).

Going forward, we have contended in section 3 that where it is necessary for the verb stem to inflect for the affix / $\mathrm{N}$-/ to impute negation, its reduplication is realized within a morphotactic structure that underscores our position of asymmetry between it and the nominal reduplication. We have noted that only the morphological stem reduplicates in 
verbal reduplication and that the affix $/ \mathrm{N}-/$ (and other verbal affixes) is inflected for only after the reduplication of the base. This is prosodically captured as [Affix-[[RED][BASE]]]. For instance, the verb-stems pra 'sweep' and sa 'fetch' are respectively negated as $\boldsymbol{m}$-pra and $\boldsymbol{n}$-sa. When reduplicated, they, respectively, become $\boldsymbol{m}$-pra-pra and $\boldsymbol{n}$-se-sa, not *m-pra-m-pra and *n-se-n-sa, suggesting the later inflection for $/ \mathrm{N}-/$. Therefore, as given in (20), we explain that, where affix $/ \mathrm{N}-/$ has to be inflected for in verbal reduplication, an alignment constraint that demands its inflection to the left of the reduplicated verb is needed; i.e. 'ALIGN $L_{[\mathrm{NEG}]}$ '. As stated in (22), this constraint will have to outrank 'MAX-IO' (and 'Truncate') in co-phonology 'Z' to optimize the morphotactic structure that will also optimally evolve the desired negated verbal reduplication; i.e. 'ALIGN $L_{[\mathrm{NEG}]}{ }{ }^{2} \mathrm{MAX}-\mathrm{IO}$ (» Truncate)'. In co-phonology ' $\mathrm{X}$ ' and co-phonology ' $\mathrm{Z}$ ', however, 'MAX-IO' outranks 'ALIGN $L_{[\mathrm{NEG}]}$ ' to ensure the morphotactic structure that will enable reduplication of base without the affix as given in (i) and (ii) of (21).

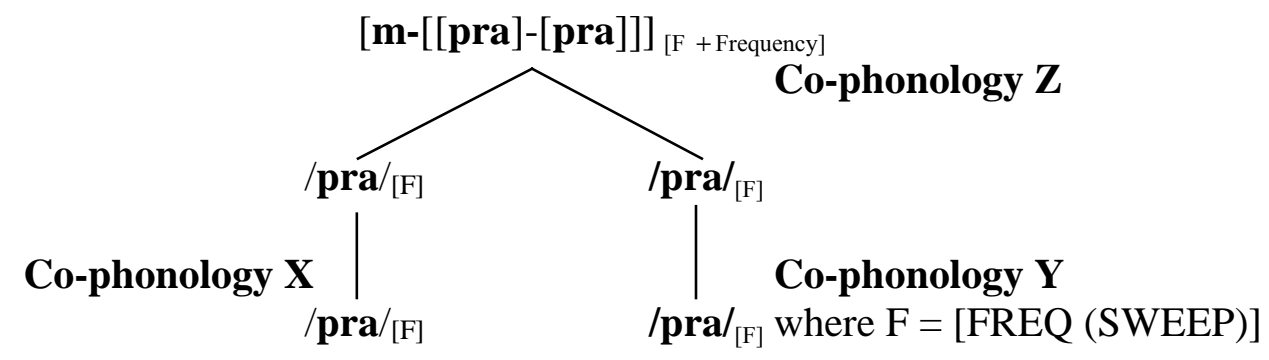

Co-phonology $X=$ MAX-IO $\gg \operatorname{ALIGN}_{[\text {NEG] }} \gg$ Truncate $_{[\text {Affix] }}$

Co-phonology $\mathrm{Y}=\mathrm{MAX}-\mathrm{IO} \gg \operatorname{ALIGN}_{[\mathrm{NEG}]} \gg$ Truncate $_{[\mathrm{Affix}]}$

Co-phonology $\mathrm{Z}=\operatorname{ALIGN}_{[\mathrm{NEG}]} \gg \mathrm{MAX}-\mathrm{IO}$ » Truncate $_{[\mathrm{Affix}]}$

\section{$\operatorname{ALIGN}_{[\mathrm{NEG}]}: \quad$ The negative affix must be at the left-edge of verbal reduplication where negation is required.}

We observe in (16) in particular that, unlike what we saw in (14), co-phonology ' $Z$ ' is different from those of ' $\mathrm{X}$ ' and ' $\mathrm{Y}$ ' and this is captured by the respective constraints rankings. This is where the morphotactic structure of the reduplicated noun forms in (17a), repeated as (23) below for ease of comparison, is asymmetrical to that of verbal reduplication in (20). That is, 


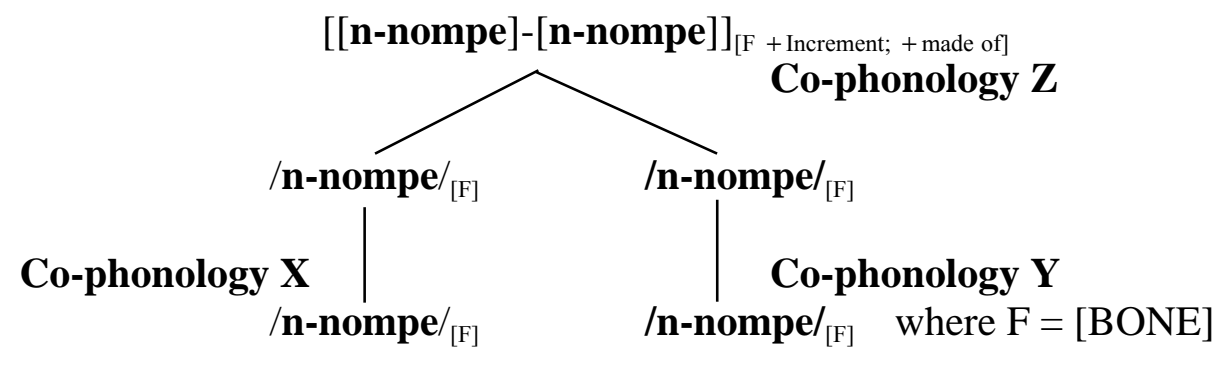

\section{Conclusion}

This paper has examined the structure of nominal and verbal reduplications in Akan. Towards observing the respective morphotactic structures of the two reduplications, it has particularly been noted that, while the stems of the two syntactic categories or constituents inflect for the affix / $\mathrm{N}$-/ for different semantic purposes in their morphological bases and even in their reduplicated forms, the morphological order of inflection for the affix between them is diverse. We have attempted to describe this diversity in inflection as an asymmetry between nominal and verbal reduplications in the language following Sato (2009). In this respect, it has been observed that, while the inflection obtains in both the base and the reduplicant in the case of the nominal reduplication, in the verbal reduplication, on the other hand, the inflection reflects in the outcome of the reduplication, such that it is only inflected for later in the reduplication and only to the left-edge of the reduplicated form. Through the Morphological Doubling Theory and with the aid of some optimality theoretic manoeuvres, where constraints and their interactions are employed, we have also captured and explained this diversity as an asymmetry between the two reduplicated forms. 
Marfo \& Osam: Optimal Inflections as Asymmetry Between Nominal and Verbal Reduplications in Akan

\section{References}

Adomako, K. 2015. "Verbal nominalization as a derivational process: The case of Akan." Ghana Journal of Linguistics 1(2): 43-64.

Appah, C. K. 2003. Nominal Derivation in Akan. MPhil Thesis submitted to NTNU, Trondheim, Norway.

Boakye, E.B. 2015. Reduplication in Akan. MPhil Thesis submitted to NTNU, Trondheim, Norway.

de Lacy, P. 1997. Prosodic categorization, MA Thesis, University of Auckland [Rutgers Optimality Archive \#236].

Dolphyne, F.A. 1988. The Akan (Twi-Fante) Language: Its Sound Systems and Tonal Structure. Accra: Ghana Universities Press.

Haspelmath, M. 2002. Understanding Morphology. London: Arnold Publishers.

Inkelas, S. 2005. "Morphological doubling theory: Evidence for morphological doubling in reduplication." In Studies in Reduplication edited by B. Hurch, 65-88. Berlin/New York: Mouton de Gruyter.

Inkelas, S. and C. Zoll. 2005. Reduplication: doubling in morphology. Cambridge: Cambridge University Press.

Marantz, A. 1982. "Rereduplication.” Linguistic Inquiry 13: 435-482.

McCarthy J. and A. Prince. 1999. "Faithfulness and identity in Prosodic Morphology." In The Prosody-Morphology Interface edited by René Kager, Harry van der Hulst and Wim Zonneveld, 218-309. Cambridge: Cambridge University Press [Excerpted in Optimality Theory in Phonology: A Reader, edited by John J. McCarthy, Malden, MA and Oxford, Blackwell (2004).]

Moravcsik, E. 1978. "Reduplicative constructions." In Universals of Human Language, Vol 3: Word Structure edited by J. Greenberg, 297-334. Stanford: Stanford University Press.

Osam E. K., C. Marfo and K. Agyekum. 2013. "The morphophonology of the Akan reduplicated word-form.” Journal of Language and Linguistic Studies, 9 (2): 45-56.

Prince A. and P. Smolensky. 2004. Optimality Theory: Constraint Interaction in Generative Grammar. Malden, MA, and Oxford: Blackwell. [Revision of 1993 technical report, Rutgers University Center for Cognitive Science. Available on Rutgers Optimality Archive, ROA-537. 
Rubino, S. 2005. "Reduplication: Form, function and distribution." In Studies in Reduplication edited by B. Hurch, 11-29. Berlin/New York: Mouton de Gruyter.

Sato, Y. 2009. "Reduplication asymmetries in Bahasa Indonesia and the organization of the lexicon-syntax interface." Journal of the Southeastern Asian Linguistics Society 1: 189-204.

Selkirk, E. 1986. “On derived domains in sentence phonology.” Phonology, 3(01): 371405.

Winkler, G. E. and S. G. Obeng. 2002. "A comparison of reduplication in Limonese Creole and Akan." In Twice as Meaningful: Morphological Reduplication in Pidgins and Creoles, edited by S. Kouwenberg, 155-171. London: Battlebridge Press. 